\title{
Antibodies to the Vi capsule of Salmonella Typhi in the serum of typhoid patients and healthy control subjects from a typhoid endemic region
}

\author{
Deborah House ${ }^{1,2}$, Vo A. Ho ${ }^{4}$,To S. Diep ${ }^{5}$, Nguyen T. Chinh ${ }^{6}$, Phan V. Bay ${ }^{4}$, Ha Vinh ${ }^{5}$, \\ Minh Duc ${ }^{4}$, Christopher M. Parry ${ }^{2,3}$, Gordon Dougan ${ }^{1}$, Nicholas J. White ${ }^{2,3}$, Jeremy J. Farrar ${ }^{2,3}$, \\ Tran Tinh Hien ${ }^{5}$, John Wain ${ }^{2,3}$ \\ ${ }^{1}$ Department of Biochemistry, Imperial College of Science, Technology and Medicine, London, United Kingdom \\ ${ }^{2}$ Centre for Tropical Medicine, Nuffield Department of Clinical Medicine, University of Oxford, Oxford, United Kingdom \\ ${ }^{3}$ Oxford University Clinical Research Unit Hospital for Tropical Diseases, Ho Chi Minh City, Viet Nam \\ ${ }^{4}$ Dong Thap Provincial Hospital, Cao Lanh, Dong Thap Province, Viet Nam \\ ${ }^{5}$ Hospital for Tropical Diseases, Ho Chi Minh City, Viet Nam \\ ${ }^{6}$ Department of Infectious Diseases, Faculty of Medicine, University of Medicine and Pharmacy, Ho Chi Minh, Viet Nam
}

\begin{abstract}
Background: There is very little published data on the antibody response to the Vi capsular polysaccharide (Vi-CPS) of S. Typhi during naturally acquired typhoid fever in an endemic area.

Methodology: An indirect ELISA, using tyraminated, purified Vi-CPS, was used to assay anti-Vi-CPS antibodies from typhoid fever cases and controls living in the Ho Chi Minh City and Mekong Delta region of Viet Nam.

Results: Antibody response to Vi-CPS is significantly higher in typhoid patients who have been ill for more than two weeks than those who are in the first two weeks of illness. The anti-Vi-CPS response is similar for adults and children. Anti-Vi-CPS antibodies can be detected in the sera of non-typhoid patients. The frequency with which this occurs increases with age, probably reflecting increased exposure to S. Typhi.

Conclusions: Anti-Vi_CPS is elicited in persons infected with S. Typhi but only after a prolonged duration of illness. Vaccine trials have shown anti-Vi-CPS antibodies to be protective; thus early treatment of typhoid patients, i.e. in the first two week of illness before the Vi-CPS response is elicited, may inhibit the development of this protective immune response.
\end{abstract}

Key Words: Vi capsule, Salmonella Typhi, typhoid fever, antibodies, Viet Nam, Vietnam

J Infect Developing Countries 2008; 2(4): 308-312.

Received 19 June 2008 - Accepted 25 June 2008

Copyright (@ 2008 House et al. This is an open access article distributed under the Creative Commons Attribution License, which permits unrestricted use, distribution, and reproduction in any medium, provided the original work is properly cited.

\section{Introduction}

Salmonella enterica sub-species enterica serovar Typhi (S. Typhi) is the causative agent of human typhoid fever. Unlike most other serovars of Salmonella enterica, S. Typhi can express a carbohydrate capsule known commonly as the $\mathrm{Vi}$ CPS antigen. The expression of this antigen is dependent upon environmental signals [1], is important for extra-cellular survival and protection against the oxidative burst of neutrophils [2], and reduces the TNF-alpha response of human macrophages after uptake. The current view is that VI-CPS is involved in immune evasion during infection of the human host [3] and is therefore of critical importance during infection.

The Vi-CPS antibody response during infection is of interest because it is protective [4] and can be used for the identification of $S$. Typhi carriers $[5,6]$.

\section{Material and Methods}

Patients and serum samples

This study was performed at the Hospital for Tropical Diseases, Ho Chi Minh City, and The Dong Thap Provincial Hospital, Cao Lanh, Dong Thap, Viet Nam using serum samples collected from children (< 15 years) and adult patients with typhoid fever and also from hospital and community control subjects. These subjects were being recruited into either treatment or epidemiology studies of typhoid fever, which have been reported elsewhere [7-9]. Serum samples from typhoid patients and hospital controls were collected either before or within the first few days of treatment and processed within a few hours of collection. Sera from community controls were 
processed within 8 hours of collection. Samples were stored at $<-20^{\circ} \mathrm{C}$ until assayed. Length of illness was taken as the time from onset of symptoms to when the sample was collected. Clinical details were recorded on a standard form. A diagnosis of typhoid fever was established by the isolation of $S$. Typhi from either bone marrow or blood.

Serum samples for the epidemiology risk factor study were collected from typhoid patients, hospital controls and community controls between May 1995 and August 1996. Hospital and community controls were recruited following the admission of a typhoid patient to the study, and were age (within five years) and sex matched with the typhoid patients. Following the recruitment of a typhoid patient, the next age-sex matched nontyphoid patient admitted to Cao Lanh Provincial Hospital was enrolled as the hospital control. Where possible patients presenting with fever were enrolled into the study, but in the absence of a suitable febrile patient a non-febrile patient was recruited. Blood cultures were performed if the patient was suspected of having typhoid fever. Diagnoses of hospital controls are given elsewhere [7]. Community controls were recruited within one month of the typhoid patient. These were recruited from the nearest household to the typhoid patient in which a suitably age-sex matched subject resided. None of the patients or controls enrolled into the study reported having been vaccinated against typhoid fever, and none of the community controls were febrile at enrolment into the study or reported having had a fever in the preceding four weeks. The median age (inter-quartile range (IQR), range) of the typhoid patients was 7 (5-14, 2-33) years compared to $6(5-15,2-39)$ years for the hospital controls and $8(5-13.5,3-39)$ years for the community controls.

Serum samples from the typhoid patients recruited to treatment trials (undertaken between 1993 and 1995) were used to compare the anti-ViCPS response of adults $(n=97)$ and children $(n=$ 63) at different stages of the disease. The median (IQR, range) in age and duration of illness was 18 $(11-26,1-63)$ years and $12(9-17,3-33)$ days, respectively.

\section{Enzyme-linked immuno-sorbent assays (ELISAs)}

In-house indirect ELISAs were established to detect anti-Vi-CPS total immunoglobulin (Ig) and
IgG. All reagents used were purchased from Sigma unless stated otherwise. The wells of the plates were washed at least three times between each step with wash buffer (saline containing $0.05 \%(v / v)$ Tween 20). Immulon $1 \mathrm{~b}$ flat bottomed 96 well microtitre plates (Dynex Technologies, Billinghurst, UK) were coated overnight at $4{ }^{\circ} \mathrm{C}$ with $50 \mu \mathrm{l}$ of either tyraminated Vi-CPS $(1 \mathrm{~g} / \mathrm{ml}$, a gift from Myron Levine, CVD, Baltimore, USA) in phosphate buffered saline (PBS, $\mathrm{pH} 7.4, \mathrm{Ag}+$ ) or PBS alone (Ag-). The plates were subsequently blocked for 1 hour at $37^{\circ} \mathrm{C}$ with $100 \mu \mathrm{l}$ PBS containing $1 \%$ bovine serum albumin (BSA). Sera were assayed at either a single dilution (1/500 for total lg, 1/250 for lgG) or serially diluted (doublingdilutions starting at a dilution of 1/50). Sera were diluted in PBS containing $0.1 \%$ BSA and $0.05 \%$ Tween $20(0.1 \%$ BSA/PBST $)$ and $50 \mu \mathrm{l}$ were applied to the appropriate wells and the plates incubated for 4 hours at room temperature. Bound antibodies were detected using heavy chainspecific goat antibodies directly conjugated to alkaline phosphatase (Sigma). These were diluted $1 / 5000$ in Tris buffered saline containing $0.1 \%$ BSA and $0.05 \%$ Tween 20 (0.1\% BSA/TBST). Fifty $\mu \mathrm{l}$ were added to each well and the plates were incubated overnight at $4^{\circ} \mathrm{C}$. Fifty $\mu \mathrm{l}$ of $\mathrm{p}$ nitrophenylphosphate $(1 \mathrm{mg} / \mathrm{ml})$ were added to each well and the plates incubated at ambient temperature in the dark for 30 to 40 minutes. The absorbance at $405 \mathrm{~nm}$ was determined using an automated ELISA reader (BioRad, Hemel Hempstead, UK).

For sera assayed at a single dilution, antibody levels are expressed in optical density (OD) units. These were taken as the mean absorbance of three wells with antigen minus the OD of a single well without antigen. A series of standards $(n=5)$ were included on each plate to which the ODs of the samples were adjusted. Inter-assay variation was monitored by the inclusion of high and low control sera on each plate. Blank wells containing serum buffer alone $(0.1 \%$ BSA-PBST) were included on each plate to monitor background. For the titration assays, sera were assayed in triplicate (two wells $\mathrm{Ag}+$, one well $\mathrm{Ag}-$ ) and the titre was taken as the highest dilution giving a net $O D$ (mean OD of $\mathrm{Ag}+$ wells minus OD of Ag- well) of $\geq$ 0.2 . Serial dilutions $(n=6)$ of a standard serum sample with a known titre were included on each plate and titre of the samples adjusted accordingly. 
Blank wells with no sera were included to monitor background.

IgG sub-class ELISAs

IgG sub-class ELISAs were performed using the above protocol but with the following modifications. The sera were assayed at $1 / 100$. Following incubation with the serum samples, 50 $\mu \mathrm{l}$ of biotinylated detecting antibody $(0.5 \mu \mathrm{g} / \mathrm{ml}$, G17-1 (IgG1) and G18-21 (IgG2), Pharmingen) were added to each well and the plates incubated overnight at $4^{\circ} \mathrm{C}$. Fifty $\mu \mathrm{l}$ of avidin-alkaline phosphatase conjugate (dilute $1 / 4000$ in $0.1 \%$ BSA/TBST) were added to each well and the plates incubated for one hour at room temperature.

\section{Statistical methods}

Serum antibody titres for the three groups of subjects were compared using paired t tests on log10 transformed data using SPSS for Windows v7.5.

\section{Results}

\section{Typhoid patients vs control}

The average antibody titres in the sera from typhoid patients were higher than those in the sera for the community and hospital controls $(p \leq 0.001$, Figure 1). For the control subjects (hospital and community) the frequency of detectable $(\geq 1 / 50)$ serum IgG titres increased with age: $1 / 47(2 \%)$ in the $2-5$ year age group; $4 / 43(9 \%)$ in the $6-10$ year age group; $13 / 40(33 \%)$ in those over 10 years. Overall the majority of patients with typhoid fever had anti-Vi-CPS IgG titres <1/50; however, most of the patients in this study were in the first week of illness.

\section{Children and adults with typhoid fever}

We were interested in looking at serum anti-ViCPS antibody levels in a larger group of typhoid patients with a longer history of illness to see if the antibody response was elicited later in the disease. Single serum samples were obtained from adults and children with non-severe typhoid fever. AntiVi-CPS total Ig and IgG levels were determined using a single dilution of the serum samples and were expressed in OD units. For cases in the first week of illness both children and adults had very low antibody levels (Figure 2a). For cases with a history of illness greater than fourteen days there was a slightly higher average antibody level in adults compared with children but this did not reach statistical significance $(p>0.05)$ because half of the adults had a very low antibody titre.

Figure 1. A comparison of Vi-CPS antibody levels (antiVi_CPS IgG) in typhoid fever cases (TF), hospitalised cases (HC) and healthy community controls (CC). Titres of $\geq 1 / 50$ were detected in sera from 22/65 (34\%) typhoid patients, 9/65 (14\%) hospital controls and 10/65 $(15 \%)$ hospital controls.

The dotted line represents the cut-off (titre of 1/50) used between raised (i.e. detectable) and undetectable levels of antibody.

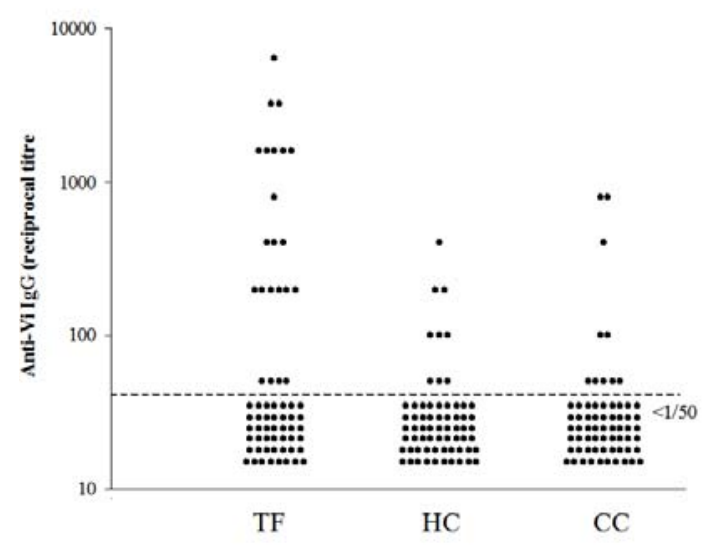

Figure 2. Boxplot of anti-Vi total lg (a) and anti-Vi lgG (b) OD values in relation to age and duration of illness. Child $\leq 14$ years, adult $>14$ years.

Solid line $=$ median, box $=$ quartile, bars = range, $\mathrm{O}$ and ${ }^{*}=$ outliers and extremes, respectively.

(a)

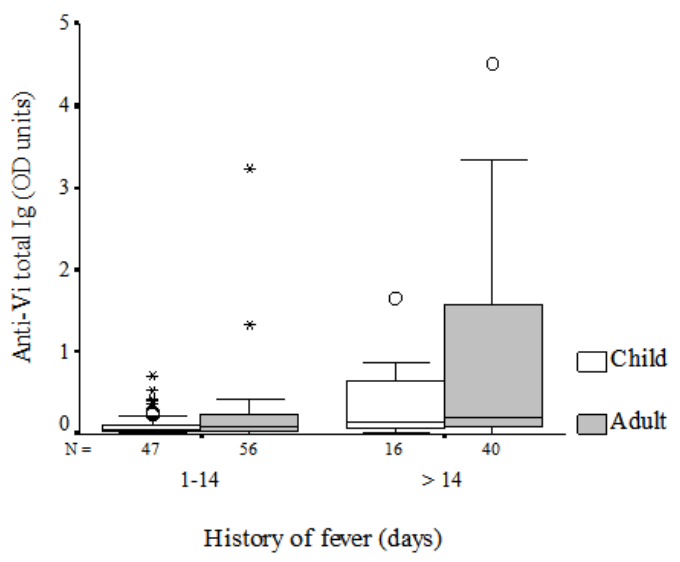


(b)

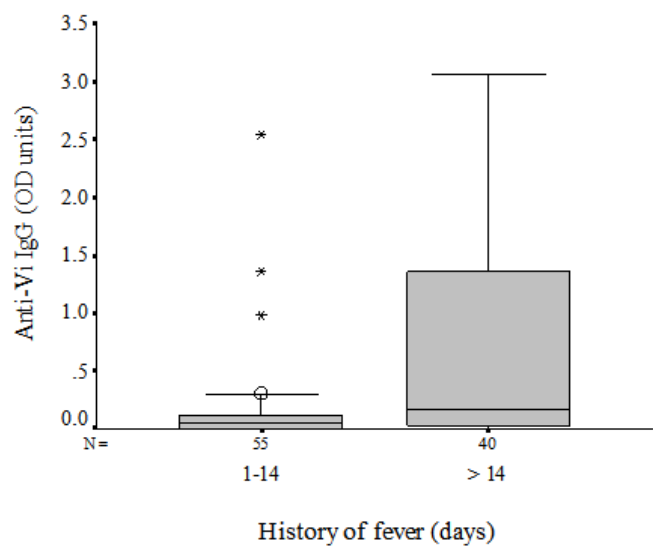

\section{Discussion}

Anti-Vi-CPS antibodies detected in serum of healthy subjects - increases with age

The data from this study show that anti-Vi-CPS antibodies can be elicited during a naturally acquired infection with $S$. Typhi, albeit late (after two weeks) in the disease and only in 35\% (23/65) of cases. Our data argue against the belief that ViCPS antibodies are not raised during an acute infection with $S$. Typhi [10]. However, the methods used here were more sensitive than those used in previous studies, which lacked sensitivity and specificity [11]. Whether or not these antibodies are protective for typhoid fever has yet to be determined but the evidence from vaccine studies shows that immunisation with purified Vi-CPS can confer protection [4]. As the Vi-CPS antigen is a T independent antigen, the protection afforded by $\mathrm{Vi}$ CPS vaccines is most likely to be antibody mediated. The same patients produced antibodies to LPS and flagella protein antigens, and so the lack of response to $\mathrm{Vi-CPS}$ is not caused by an inability to produce antibody [7], nor is it caused by the failure of S. Typhi to express Vi-CPS in vivo. In Viet Nam, as in Pakistan, over $99 \%$ of clinical isolates of S. Typhi express the Vi-CPS [12]. This raises the question as to whether the prompt diagnosis and treatment of an S. Typhi infection is conducive to the development of protective immunity, or whether patients successfully treated in hospital should be immunised before being discharged. Vi-CPS, however, is not the only antigen to which protective antibodies can be raised. A live attenuated $S$. Typhi vaccine, Ty21a, does not express the Vi-CPS antigen but is an effective vaccine, even giving some cross protection against S. Paratyphi B infection [13].
In this study, raised anti-Vi-CPS IgG titres were seen in 10/65 of the non-typhoid control subjects. This may be due to exposure to the antigen over time or it may indicate carriage of $S$. Typhi. Unfortunately we were unable to determine carrier status because the serum samples were processed some time after the sample collection, and stool culture on the subjects with raised titres was not possible. Although the numbers of sera tested in this present study were relatively small, our results are similar to the findings of others in typhoid endemic regions [14, 15]; there is an increase in Vi-CPS antibody levels with age.

By the end of the second week of illness, antiVi-CPS antibodies are produced by approximately one third of acute cases of typhoid fever. There is very little difference in the antibody response between adults and children but this may be masked by the two thirds of cases who did not produce an antibody response to Vi-CPSa antigen.

\section{Acknowledgements}

All studies were approved by the Scientific and Ethical Committee of the participating institutions and informed consent was obtained from all participants or, in the case of children, from their parent or guardian.

\section{References}

1. Pickard D et al. (1994) Characterization of defined ompR mutants of Salmonella typhi: ompR is involved in the regulation of Vi polysaccharide expression. Infect Immun 62(9):3984-3993.

2. Looney RJ and Steigbigel RT (1986) Role of the Vi antigen of Salmonella typhi in resistance to host defense in vitro. J Lab Clin Med 108(5):506-516.

3. Raffatellu M et al. (2006) Capsule-mediated immune evasion: a new hypothesis explaining aspects of typhoid fever pathogenesis. Infect Immun 74(1):19-27.

4. Arya SC and Agarwal N (2008) Re: typhoid fever vaccines: systematic review and meta-analysis of randomised clinical trials. Vaccine26(3):291.

5. Nolan CM et al. (1980) Evaluation of a new assay for $\mathrm{Vi}$ antibody in chronic carriers of Salmonella typhi. 12(1):2226.

6. Losonsky GA et al. (1987) Development and evaluation of an enzyme-linked immunosorbent assay for serum $\mathrm{Vi}$ antibodies for detection of chronic Salmonella typhi carriers.. 25(12):2266-2269.

7. House D et al. (2001) Serology of Typhoid Fever in an Area of Endemicity and Its Relevance to Diagnosis. J Clin Microbiol 39(3):1002-7.

8. Luxemburger C et al. (2001) Risk factors for typhoid fever in the Mekong delta, southern Viet Nam: a case-control study. Trans R Soc Trop Med Hyg 95(1):19-23.

9. Parry CM et al. (2007) Randomized controlled comparison of ofloxacin, azithromycin, and an ofloxacinazithromycin combination for treatment of multidrug- 
resistant and nalidixic acid-resistant typhoid fever. Antimicrob Agents Chemother 51(3):819-25.

10. Lin FY et al. (1988) Restaurant-associated outbreak of typhoid fever in Maryland: identification of carrier facilitated by measurement of serum Vi antibodies. J Clin Microbiol. 26(6):1194-7.

11. Edelman R and Levine MM (1986) Summary of an international workshop on typhoid fever. Rev Infect Dis. 8(3):329-349.

12. Wain J et al. (2005) Vi antigen expression in Salmonella enterica serovar Typhi clinical isolates from Pakistan. J Clin Microbiol 43(3):1158-65.

13. Levine MM et al. (2007) Ty21a live oral typhoid vaccine and prevention of paratyphoid fever caused by Salmonella enterica Serovar Paratyphi B Clin Infect Dis 45 Suppl 1:S24-8.

14. Klugman KP et al. (1987) Protective activity of Vi capsular polysaccharide vaccine against typhoid fever. Lancet 2(8569):1165-1169.

15. Keddy $\mathrm{KH}$ et al. (1999) Persistence of antibodies to the Salmonella typhi Vi capsular polysaccharide vaccine in South African school children ten years after immunization. Vaccine. 17(2):110-3.

Corresponding Author: John Wain, Wellcome Trust Sanger Institute, Hinxton Cambs. CB10 1SA. Email jw5@sanger.ac.uk

Conflict of interest: No conflict of interest is declared. 